\title{
Minimizing sample shape and size effects in all purpose magnetometers
}

\author{
J. T. Van der Noordaa ${ }^{a)}$ \\ Department of Physics, California State University, Fresno, California 93740, USA
}

(Received 14 March 2008; accepted 30 November 2008; published online 7 January 2009)

\begin{abstract}
When measurements are made with a magnetometer, it is usually assumed that the sample produces a pure dipolar magnetic field. This is only true if the sample is a sphere. If the sample is not a sphere, the magnetic field produced by it will also contain higher order multipoles. These components of the magnetic field depend not only on the volume and magnetization but also on the sample's shape and size. Ideally the measured moment of a sample is only proportional to the sample's magnetization and volume. This paper explains why a sample's shape and size affects the response of a magnetometer, shows results illustrating this effect, and discusses conditions that will minimize these effects. () 2009 American Institute of Physics. [DOI: 10.1063/1.3053418]
\end{abstract}

\section{INTRODUCTION}

A sensitive balance is affected by wind currents. No one calculates the effect of the wind, rather the balance is enclosed in a box, eliminating the wind and the need to correct for it. When the magnetic moment of a sample is measured, a similar problem can occur. The measurement may be influenced by the shape and size of the sample. This paper explains why a sample's shape and size can influence the measured moment of the sample and how experimenters can avoid having to apply corrections (an especially difficult task for irregularly shaped samples). For some measurements, with cylindrical samples of precisely known dimensions, ${ }^{1}$ the best approach is to determine the susceptibility from the mutual inductance $e^{2,3}$ (these references also show that cylindrical sample size effects are well known). In general, the magnetic field produced by a magnetized sample can be described in terms of a multipole expansion starting with a dipole term. Only spherical samples produce a pure dipolar magnetic field (the only part directly proportional to the magnitude of the magnetic moment). The pickup coil flux, due to a large nonspherical sample, will also depend on higher order multipoles of the magnetic field. These higher order terms depend on shape and size factors describing the spatial distribution of the magnetic moment. This is illustrated below by exam- ining the magnetic field produced by a spheroidal sample (the simplest nonspherical shape for which exact results can be developed). Included is a multipole expansion of the magnetic field created by a cylindrical sample (although spheres can be better, cylinders are often used for calibration). ${ }^{4} \mathrm{~A}$ useful procedure is introduced to determine the appropriate size of a sample.

\section{MAGNETIC FIELD INSIDE AND OUTSIDE A MAGNETIZED PROLATE SPHEROID}

The effect of sample shape and size on magnetometer response may be shown by considering a prolate spheroidal sample, as shown in Fig. 1. The magnetic field produced by this sample, magnetized by an external magnetic field $\vec{H}_{a}$ can be calculated by using a magnetic potential $V_{m}$ and the Laplace equation in prolate spheroidal coordinates. These are the variables $\eta$ (constant on a prolate spheroidal surface), $\xi$ (constant on each sheet of a two-sheet hyperboloid), and the familiar azimuthal angle $\phi$. The general solution is an expansion of Legendre polynomials of the first and second kind. Boundary conditions are applied at the origin, the surface of the sample, and at infinity. The resulting magnetic potential is $^{5}$ (in SI units)

$$
V_{m}(\eta, \xi)= \begin{cases}-H_{a} \frac{c}{D \chi+1} \eta \xi, & \eta<\frac{a}{c} \\ H_{a}\left[-c \eta+\frac{\chi b^{2} a}{c^{2}(D \chi+1)}\left\{\frac{1}{2} \eta \ln \left(\frac{\eta+1}{\eta-1}\right)-1\right\} \xi\right], & \eta>\frac{a}{c} .\end{cases}
$$

Here $c^{2}=a^{2}-b^{2}$ ( $a$ and $b$ are the spheroid's semiaxes, see Fig. 1), $D$ is the demagnetization factor, ${ }^{6}$ and the susceptibility ${ }^{7} \chi$ equals the magnetization divided by the field inside the sample.

Taking the gradient and converting to cylindrical coordinates, ${ }^{8}$ one finds that the sum of the external field and the field produced by the magnetized sample equals

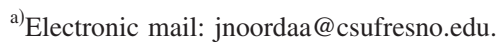




$$
\vec{H}(z, \rho)=H_{a} \begin{cases}\frac{\hat{z}}{D \chi+1}, & \text { inside } \\ \hat{z}+\frac{\chi b^{2} a}{c^{3}(D \chi+1)} \sum_{\ell=1} \frac{1}{2 \ell+1}\left(\frac{c}{r}\right)^{2 \ell+1}\left[2 \ell P_{2 \ell}(z / r) \hat{z}+P_{2 \ell}^{1}(z / r) \hat{\rho}\right], & \text { outside. }\end{cases}
$$

Here $r=\sqrt{\rho^{2}+z^{2}}$. The Legendre polynomials and the associated Legendre polynomials are denoted by $P_{2 \ell}(z / r)$ and $P_{2 \ell}^{1}(z / r)$, respectively (signs as in Ref. 5). The components of the magnetic field were determined on the $z$ axis $^{9}$ and then matched to a Legendre polynomial expansion. For long prolate spheroids, replace $c / r$ by $r / c$ where $c>r$ outside the sample. This formula is also correct for oblate spheroids; it is easier to write the expansion in terms of $d^{2}=b^{2}-a^{2}$ and use the oblate spheroid demagnetization factor ( $c$ is imaginary).

Pickup coils wound in opposition only measures the sample's magnetic field. The $z$ component of the magnetic field produces the flux when the pickup coil loops are parallel to the $x-y$ plane. This component of the magnetic induction outside the sample of volume $V$ equals

$$
B_{z}^{\text {out }}(\vec{r})=\frac{\mu_{0}}{2 \pi} V M \sum_{\ell=1}^{\infty} \frac{A_{2 \ell}}{r^{2 \ell+1}} P_{2 \ell}(z / r),
$$

where $A_{2 \ell}=(3 \ell /(2 \ell+1)) c^{2 \ell-2}$ and $M$ is the magnetization. For spheres only $A_{2}$ is nonzero. The relation between the magnetization and the three component parameters $(\chi, D$, and $H_{a}$ ) in Eq. (2) is

$$
\frac{H_{a}}{M}=\frac{1}{\chi_{\mathrm{ex}}}=D+\frac{1}{\chi} \text {. }
$$

This equation defines the external susceptibility $\chi_{\mathrm{ex}}$.

\section{MAGNETIC FIELD PRODUCED BY A MAGNETIZED CYLINDER}

The magnetic field produced by a magnetized cylinder in a uniform field can be approximated by assuming that the

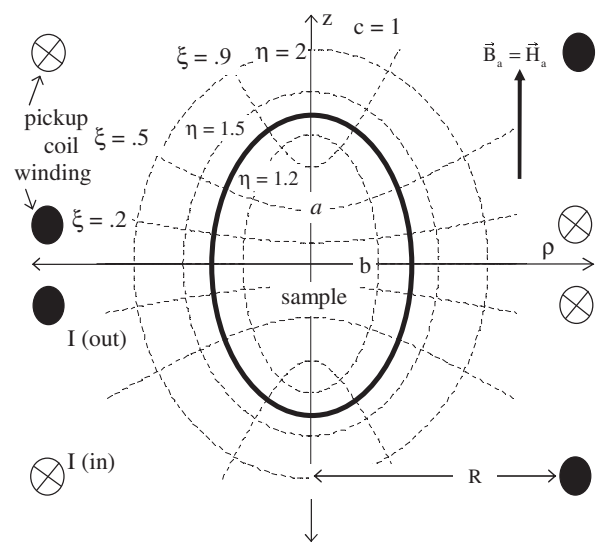

FIG. 1. A prolate spheroidal magnetic sample with axes $a$ and $b$ in a uniform magnetic field inside a second derivative pickup coil of radius $R$ (the direction of the current $I$ shows the sense of the windings). Spheroidal (dashed) and cylindrical (solid) coordinates are shown. The bold ellipse denotes the surface of the sample. magnetization inside is uniform. The cylinder ends then have a uniform "surface charge" equal to the magnetization $M$. An infinitesimal cylindrical element with a cross section of $d A$ and height $2 a$ produces a magnetic potential

$$
V_{m}^{d A}(\vec{r})=\frac{M d A}{\pi r} \sum_{\ell=0}^{\infty} \ell\left(\frac{a}{r}\right)^{2 \ell+1} P_{2 \ell+1}(z / r)
$$

The $z$ component of the field produced on the $z$ axis by a magnetized cylinder is found by taking the gradient of this potential and integrating over the cylinder's cross sectional area (of radius $b$ ),

$$
H_{z}^{\mathrm{out}}(z)=-2 M \sum_{\ell=1}^{\infty} \ell\left(\frac{a}{z}\right)^{2 \ell-1} \int_{1}^{z / \sqrt{z^{2}+b^{2}}} d y y^{2 \ell-2} P_{2 \ell}(y) .
$$

After integrating, the expansion is sorted in powers of $1 / z$ $\left(1 / z^{3}, 1 / z^{5}, 1 / z^{7}\right.$, etc. $)$. When the sorted expression is matched to a Legendre polynomial expansion on the $z$ axis, the magnetic induction can be expressed as Eq. (3) using the $A_{2 \ell}$ coefficients of Appendix A. Integrating the expression for a current loop ${ }^{10}$ over the length of a coil, the size of the cylinder gives the same result.

\section{MAGNETIC FLUX PRODUCED BY A SAMPLE IN A PICKUP COIL}

The total flux in a pickup coil is the sum of the contributions of each of the loops,

$$
\Phi_{\text {tot }}=\sum_{\text {loop }} \Phi_{\text {loop }}=\frac{\mu_{\mathrm{o}} M V}{R} \sum_{\text {loop }}\left[\sum_{\ell=1}^{\infty} \frac{A_{2 \ell}}{R^{2 \ell-2}} s_{2 \ell}\left(\frac{z_{\text {loop }}}{R}\right)\right],
$$

where $R$ is the pickup coil radius, $z_{\text {loop }}$ is the distance from the loop to the sample's center, and

$$
s_{2 \ell}(x)=-\int_{1}^{\infty} \frac{d u u}{\left(u^{2}+x^{2}\right)^{(\ell+(1 / 2))}} P_{2 \ell}\left(\frac{x}{\sqrt{u^{2}+x^{2}}}\right) .
$$

These dimensionless functions describe the flux produced by each multipole term of the magnetic field in each loop (Appendix $\mathrm{B}$ has the lowest order functions). Because $\nabla \cdot \vec{B}=0$ it is possible and easier to integrate outside the pickup coil (from 1 to $\infty$ ). For spheroidal samples Eq. (7) is well behaved as long as $\left|c^{2} / R^{2}\right|<1$. The behavior of the cylindrical $A_{2 \ell}$ coefficients cannot be determined in terms of a single parameter, but $a$ must be less than $R$ in order for the approximation to be valid [see Eq. (5)]. If sections of the pickup coil have different radii (say, $R_{1}$ and $R_{2}$ ), then Eq. (7) will have two parts (for $z_{\text {loop }} / R_{1}$ and for $z_{\text {loop }} / R_{2}$ ).

For nonspherical samples a useful figure of merit is the factor $\Delta=-\frac{3}{10} c^{2} / R^{2}$ (the ratio of the second and first terms of 


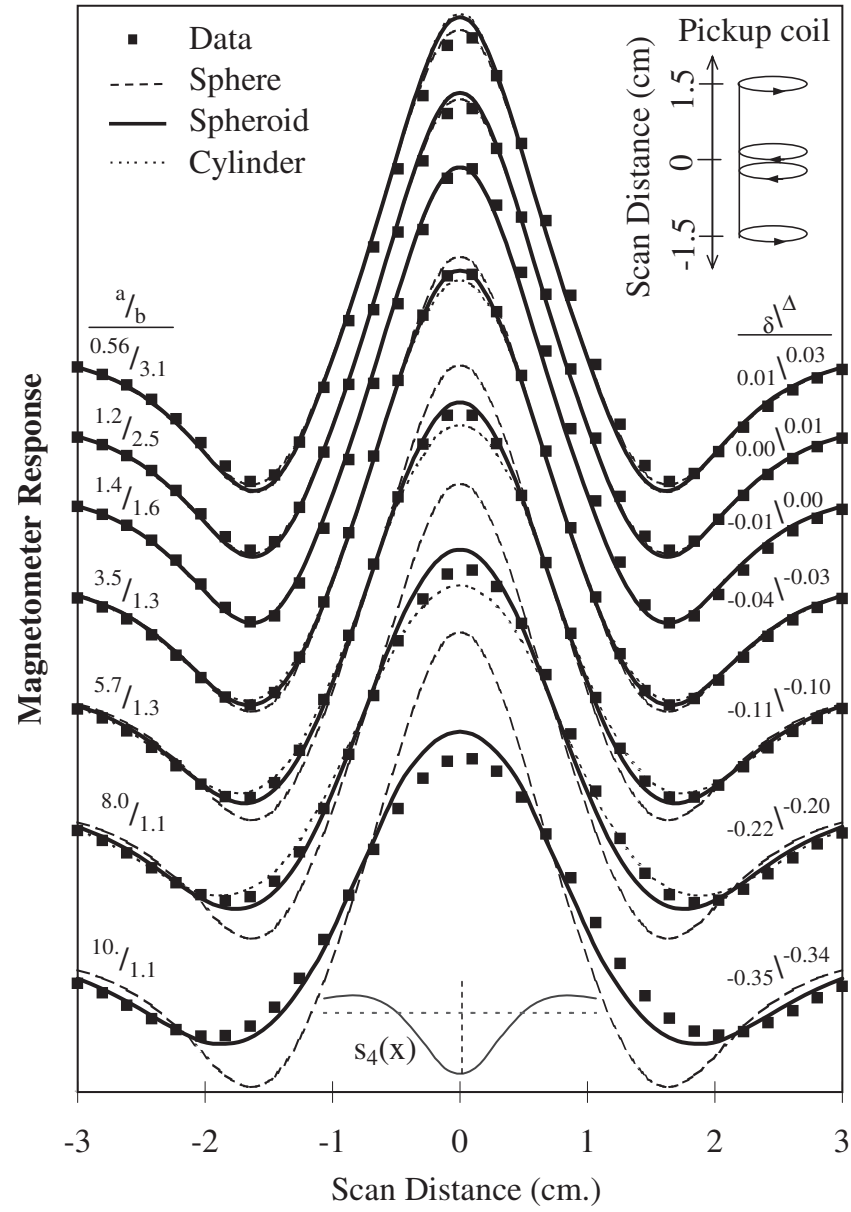

FIG. 2. Magnetometer response as a function of scan distance. Samples are labeled on the left by sample dimensions $a$ and $b$ (in millimeter) and on the right by actual $(\delta)$ and estimated $(\Delta)$ deviations (see text). The uncertainty in the data is about $1 \%-2 \%$.

Eq. (7) for the spheroidal case with one loop at $z_{\text {loop }}=0$ ). The amount by which a sample is out of round is given by $c^{2}$ and $\Delta$ measures the fraction of flux produced by the $1 / r^{5}$ term compared to the $1 / r^{3}$ term.

\section{EXPERIMENTAL DEMONSTRATION AND CONCLUSION}

Figure 2 shows representative response curves of a Quantum Design magnetic property measurement system magnetometer when various samples of $\mathrm{Mn} \cdot\left(\mathrm{NH}_{4}\right)_{2}\left(\mathrm{SO}_{4}\right)_{2} \cdot 6 \mathrm{H}_{2} \mathrm{O}$ (MAS) are moved through a pickup coil (drawn in the upper right of Fig. 2, $R$ $=0.97 \mathrm{~cm}$.) Curie constants were determined for each sample using data at 5, 10, 15, and $20 \mathrm{~K}$ in three magnetic fields $(0.01,0.03$, and $0.05 \mathrm{~T})$. Moments were calculated by magnetometer software assuming dipole behavior. MAS obeys the Curie-Weiss law at these temperatures with a $\mathrm{Cu}$ rie constant of $1.66 \times 10^{-3} \mathrm{~K}$. Figure 2 contains representative raw data (at $5 \mathrm{~K}$ and $0.05 \mathrm{~T}$ ) divided by the sample mass and adjusted for the gain. ${ }^{11}$ The left side of Fig. 2 gives $a$ and $b$ in millimeter of the roughly carved, cylindricallyspheroidally shaped samples. The measured deviation $\delta$ and the figure of merit $\Delta$ are written on the right side of Fig. 2. For example, the measured Curie constant of the sample $a / b=\frac{8.0}{1.1}$ is $22 \%$ too low $(\delta=-0.22)$ and the $1 / r^{5}$ term for a pickup loop at $z=0$ reduces the flux of the $1 / r^{3}$ term by $20 \%$ $(\Delta=-0.20)$. In Fig. 2 all the spherical theory curves have the same height (adjusted to match sample $\frac{1.4}{1.6}$ ). There are no additional adjustments for the spheroidal and cylindrical curves (which have terms up to $2 \ell=14$ ). Equation ( 7 ) is no longer valid for $\frac{10}{1.1}(a>R)$ and a separate expression for the spheroidal theory was used (containing terms for $c^{2} / r^{2}$ and $r^{2} / c^{2}$ up to $2 \ell=60$ ). The difference in response for the samples is due to the difference in sample shapes and sizes. The mass (volume) of the samples varied also, but this does not explain the difference in response (for example, sample $\frac{1.2}{2.5}$ was twice as massive as $\frac{1.4}{1.6}$, but the Curie constant varied by only a percent). ${ }^{12}$ Figure 2 clearly shows that if the sample is too prolate, the magnetometer response is much different from that of a point dipole response.

Usually the goal is to measure the susceptibility ${ }^{13} \chi$, and it depends on $D$ [see Eq. (4)]. As discussed previously, ${ }^{14}$ for certain measurements it is better to have very prolate samples. The relative uncertainty in the susceptibility $\left(\sigma_{\chi}\right)$ depends linearly on the susceptibility as well as the relative uncertainties in $\chi_{\mathrm{ex}}\left(\sigma_{\chi_{\mathrm{ex}}}\right)$ and $D\left(\sigma_{D}\right)$,

$$
\sigma_{\chi}=\sqrt{\left(\sigma_{\chi_{\mathrm{ex}}} \chi / \chi_{\mathrm{ex}}\right)^{2}+\left(\sigma_{D} D \chi\right)^{2}} .
$$

The most sensitive measurement of $\chi_{\mathrm{ex}}$ is made with a spherical sample of radius $R$ and volume $V_{R}$. The volume of any spheroidal sample, $V=(4 \pi / 3) a\left(a^{2}-c^{2}\right)$, is a fraction $\nu$ of $V_{R}\left(V=\nu V_{R}\right)$. Using the approximation $c^{2}=-3 \Delta R^{2}$ and solving the spheroidal volume equation for $a$ give

$$
a=R\left\{\left(\nu / 2+\sqrt{\nu^{2} / 4-\Delta^{3}}\right)^{1 / 3}+\left(\nu / 2-\sqrt{\nu^{2} / 4-\Delta^{3}}\right)^{1 / 3}\right\} .
$$

Once acceptable values for $\Delta$ and $\nu$ are established, the allowed range for $a$ can be found. For example, imagine that $\Delta=0.01$ and $\nu=0.01$ are acceptable. Using Eq. (10) then gives $a_{\max }=0.26 R(a / b=1.3)$ and $a_{\min }=0.17 R(a / b=0.7)$. If the sample is too big, its dimensions will have to be trimmed to keep $\Delta$ to 0.01 . If the sample is a soft ferromagnet with $a / b=1.3$, then near the critical temperature $T_{c}, \chi_{\mathrm{ex}} \approx 1 / D$ $\approx 3.8$ and $\chi_{\mathrm{ex}}$ is small compared to $\chi$ [see Eq. (4)]. The relative error in the susceptibility $\sigma_{\chi}$ may be too large. Suppose $\sigma_{\chi}$ remains sufficiently small when $\nu$ is lowered to $1 \times 10^{-5}$, then $a_{\max }=0.17 R(a / b=22)$ and $a_{\min }=3.3$ $\times 10^{-5} R\left(a / b=1.9 \times 10^{-3}\right)$. Now $\chi_{\mathrm{ex}} \approx 1 / D \approx 180$ (for $a / b$ =22) near $T_{c}$ and $\sigma_{\chi}$ would decrease by a factor of 50. The agreement between $\Delta$ and $\delta$ is good because the higher order terms drop off rapidly and $70 \%$ of the flux in a long coil comes from windings with $z \leq R$ [integrate $s_{2}(z / R)$ to see this].

Equation (7) can always be used to determine sample shape and size effects more accurately. This equation is also useful for designing strategies to limit these effects. Figure 2 shows that the $1 / r^{5}$ term does not contribute to the flux of loops at $z / R= \pm 0.5$ because $s_{4}( \pm 0.5)$ is zero. Excluding data within $0.4 \mathrm{~cm}$ of each peak (at $-1.5,0$, and $1.5 \mathrm{~cm}$ ) lowers $\delta$ for sample $\frac{8.0}{1.1}$ from -0.22 to -0.04 [the change is less for $\frac{10}{1.1}$ since its $1 / r^{7}$ term is large and $\left.s_{6}( \pm 0.5) \neq 0\right]$. Experiment and theory do agree more outside the peaks (see 
Fig. 2). For this magnetometer, one can compare the analysis of off-peak data with that of all the data to see if shape effects are experimentally significant. In magnetometers where samples are fixed in position, pickup loops can be strategically placed to limit shape and size effects. The $1 / r^{5}$ term is small for a fixed sample in one of two opposing $6 R$ long coils since $s_{4}(x) \approx 0$ for $|x|>3$ and $s_{4}(x)$ integrates nearly to zero.

\section{ACKNOWLEDGMENTS}

Linton R. Corruccini and an anonymous reviewer made very helpful comments. Robert Shelton and Peter Klavins made their magnetometers available for the experimental work.

\section{APPENDIX A: COEFFICIENTS FOR DETERMINING THE FIELD PRODUCED BY A CYLINDRICAL SAMPLE}

$$
\begin{aligned}
A_{2}= & 1, \quad A_{4}=2 a^{2}-\frac{3}{2} b^{2}, \\
A_{6}= & 3 a^{4}-\frac{15}{2} a^{2} b^{2}+\frac{15}{8} b^{4}, \\
A_{8}= & 4 a^{6}-21 a^{4} b^{2}+\frac{35}{2} a^{2} b^{4}-\frac{35}{16} b^{6}, \\
A_{10}= & 5 a^{8}-45 a^{6} b^{2}+\frac{315}{4} a^{4} b^{4}-\frac{525}{16} a^{2} b^{6}+\frac{315}{128} b^{8}, \\
A_{12}= & 6 a^{10}-\frac{165}{2} a^{8} b^{2}+\frac{495}{2} a^{6} b^{4}-\frac{3465}{16} a^{4} b^{6}+\frac{3465}{64} a^{2} b^{8} \\
& -\frac{693}{256} b^{10}, \\
A_{14}= & 7 a^{12}-\frac{273}{2} a^{10} b^{2}+\frac{5005}{8} a^{8} b^{4}-\frac{15015}{16} a^{6} b^{6}+\frac{63063}{128} a^{4} b^{8} \\
& -\frac{21021}{256} a^{2} b^{10}+\frac{3003}{1024} b^{12}
\end{aligned}
$$

\section{APPENDIX B: LOWEST ORDER LOOP FLUX FUNCTIONS}

$$
\begin{aligned}
& s_{2}(x)=\frac{1}{2}\left(1+x^{2}\right)^{-3 / 2}, \quad s_{4}(x)=\left(\frac{1}{2} x^{2}-\frac{1}{8}\right)\left(1+x^{2}\right)^{-7 / 2} \\
& s_{6}(x)=\left(\frac{1}{2} x^{4}-\frac{3}{4} x^{2}+\frac{1}{16}\right)\left(1+x^{2}\right)^{-11 / 2}
\end{aligned}
$$

$$
\begin{aligned}
s_{8}(x)= & \left(\frac{1}{2} x^{6}-\frac{15}{8} x^{4}+\frac{15}{16} x^{2}-\frac{5}{128}\right)\left(1+x^{2}\right)^{-15 / 2}, \\
s_{10}(x)= & \left(\frac{1}{2} x^{8}-\frac{7}{2} x^{6}+\frac{35}{8} x^{4}-\frac{35}{32} x^{2}+\frac{7}{256}\right)\left(1+x^{2}\right)^{-19 / 2}, \\
s_{12}(x)= & \left(\frac{1}{2} x^{10}-\frac{45}{8} x^{8}+\frac{105}{8} x^{6}-\frac{525}{64} x^{4}+\frac{315}{256} x^{2}-\frac{21}{1024}\right) \\
& \times\left(1+x^{2}\right)^{-23 / 2}, \\
s_{14}(x)= & \left(\frac{1}{2} x^{12}-\frac{33}{4} x^{10}+\frac{495}{16} x^{8}-\frac{1155}{32} x^{6}+\frac{3465}{256} x^{4}-\frac{693}{512} x^{2}\right. \\
& \left.+\frac{33}{2048}\right)\left(1+x^{2}\right)^{-27 / 2} .
\end{aligned}
$$

${ }^{1}$ Demagnetization factors are in D.-X. Chen, J. A. Brug, and R. B. Goldfarb, IEEE Trans. Magn. 27, 3601 (1991).

${ }^{2}$ R. B. Goldfarb and J. B. Minervini, Rev. Sci. Instrum. 55, 761 (1984), before 1995 Quantum Design had data.

${ }^{3}$ D.-X. Chen, Meas. Sci. Technol. 15, 1195 (2004).

${ }^{4} \mathrm{See} \quad \mathrm{http} / /$ ts.nist.gov/MeasurementServices/ReferenceMaterials/ archived_certificates.cfm, SRM 765.

${ }^{5}$ G. Arfken, Mathematical Methods for Physicists (Academic, New York, 1970); J. A. Stratton, Electromagnetic Theory, (McGraw-Hill, New York, 1941) shows how to do this calculation.

${ }^{6} D=b^{2} a / c^{3}\{\ln [(a+c) /(a-c)] / 2-c / a\}$ see J. A. Osborn, Phys. Rev. 67, 351 (1945).

${ }^{7}$ For paramagnetic (diamagnetic) samples $\chi$ is positive (negative) and for type I superconductors $\chi=-1$.

${ }^{8}$ Make the conversion with $c \eta \xi=z$ and $\eta^{2}=\left(\rho^{2}+z^{2}+c^{2}\right.$

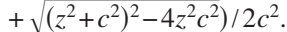

${ }^{9}$ The $\rho$ component is actually zero on the $z$ axis but $r H_{\rho} / \rho$ is not zero on the $z$ axis and one proceeds from there.

${ }^{10}$ J. D. Jackson, Classical Electrodynamics, 2nd ed. (Wiley, New York 1975), p. 177.

${ }^{11}$ See http://www.qdusa.com/resources/pdf/mpmsappnotes/1014-213.pdf for parameters Quantum Design uses.

${ }^{12}$ Varying mass by $180 \%$ of rough spheres fixed in a coil with halves wound in opposition was not significant either.

${ }^{13}$ Or the local susceptibility $\chi_{\text {local }}, M$ divided by $H$ at a magnetic ion site, see A. H. Cooke, D. A. Jones, J. F. A. Silva, and M. R. Well, J. Phys. C 8, 4083 (1975).

${ }^{14}$ D.-X. Chen, E. Pardo, and A. Sanchez, IEEE Trans. Magn. 41, 2077 (2005) 